\title{
Bronchoscopic Diagnosis of Peripheral Lung Lesions
}

\author{
Stephen Lam ${ }^{a}$ Pallav L. Shah ${ }^{b, c, d}$ \\ aDepartment of Integrative Oncology, British Columbia Cancer Research Institute and Department of Medicine, \\ University of British Columbia, Vancouver, BC, Canada; ${ }^{b}$ Department of Respiratory Medicine, Royal Brompton \\ Hospital, London, UK; ' ${ }^{2}$ epartment of Respiratory Medicine, Chelsea \& Westminster NHS Foundation Trust, London, \\ UK; ${ }^{d}$ National Heart and Lung Institute, Imperial College London, London, UK
}

Following the finding in the National Lung Screening Trial in the USA and the Dutch-Belgian NELSON trial in Europe that lung cancer screening with lowdose computed tomography can reduce lung cancer by $20-24 \%$ in high-risk smokers, lung cancer screening has been implemented around the world $[1,2]$. Sixty to eighty percent of the lung cancers found on the baseline screening low-dose computed tomography are $\leq 2 \mathrm{~cm}$ [3-5]. In repeat screening, $76-87 \%$ of the lung cancers are $\leq 2 \mathrm{~cm}[4,6,7]$. With an average diagnostic yield of $34 \%$, conventional flexible bronchoscopy techniques have a limited role in the evaluation of peripheral pulmonary nodules $\leq 2 \mathrm{~cm}$ [8]. To improve the accuracy of bronchoscopic diagnosis, virtual bronchoscopy with or without electromagnetic navigation (EMN) to guide insertion of the bronchoscope toward the target lesion, thin or ultrathin bronchoscopes to visualize the lesion or get as closed to the lesion as possible, and confirmation of arrival at the target before tissue sampling using radial endoscopic ultrasound (R-EBUS), fluoroscopy, $\mathrm{CT}$, cone beam CT or a combination of R-EBUS, and radiology imaging are used. A recent meta-analysis of

karger@karger.com

(c) 2021 S. Karger AG, Basel

www.karger.com/res

Karger
R-EBUS-guided bronchoscopy showed a pooled sensitivity of 0.72 (95\% CI $0.70-0.75)$ [9]. There was no association between sensitivity and the use of virtual bronchoscopy or fluoroscopy. Another meta-analysis comparing the performance of R-EBUS and EMN showed a similar diagnostic accuracy for malignant nodules between the 2 techniques; $72.4 \%$ (95\% CI 68.776.1 for R-EBUS) and $76.4 \%$ (95\% CI 70.8-82.0 for EMN) [10]. The role of virtual bronchoscopy, R-EBUS, fluoroscopy-guided bronchoscopy using a $3.0-\mathrm{mm}$ ultrathin bronchoscope without a guide sheath (GS) versus a $4.0-\mathrm{mm}$ thin bronchoscope with a GS was compared in a randomized trial. The diagnostic yield was significantly higher in the ultrathin bronchoscope group than that in the thin bronchoscope group (70.1 vs. $58.7 \%$, respectively; $p=0.027$ ) [11]. More recently, robotic-assisted bronchoscopy techniques have been associated with improved reach of target lesions. Successful localization with R-EBUS was 96\%. However, the diagnostic yield for concentric lesion was $80.6 \%$; for eccentric lesion, it was 70\% [12]. The discrepancy between the successful target localization rate of over $90 \%$ 
and a diagnostic yield of $70-80 \%$ in the R-EBUS and robotic bronchoscopy studies have not been adequately explained.

The bronchoscopy-pathology correlation study by Fukada and colleagues [13] reported in this issue provides an explanation as to why tissue sampling after arrival at the target lesion with confirmation by R-EBUS may not yield a diagnosis. The investigators used a physiological saline injection technique to dilate the small peripheral airways, leading the tumor to allow clearer observation of the endo-luminal features and then classified them into a stenotic or nonstenotic type and to determine if the tumor was exposed on the surface or submucosal with an intact ciliated epithelium as reflected by a light blue line using narrow band imaging. The latter cannot be done using a 3-mm ultrathin bronchoscope, but an intact epithelium can be recognized on white light examination. As can be expected, the diagnostic yield of bronchial brush and bronchial wash cytology was much lower with the nonexposed tumor types, most of them being adenocarcinomas. The ability to visualize the tumor using an ultrathin bronchoscope to take a biopsy using forceps or TBNA explains the better diagnostic yield than a "blind" biopsy using a GS after removal of the R-EBUS probe [11]. The R-EBUS image can appear to be concentric or eccentric, but the biopsy can be nondiagnostic when the tumor is of the nonexposed type, especially if the airway is nonstenotic. In a robotic bronchoscopy study where a flexible 19- to 23-G needles was used for transbronchial needle aspiration along with conventional biopsy tools where the needle can sample submucosal or peribronchial lesions, the difference between reaching the target lesions and a positive diagnosis of malignancy was smaller - 97 and $88 \%$, respectively [14]. The alternative is to access the nodule by penetrating through the bronchus at an appropriate location and traversing the lung parenchyma to sample the nodule $[15,16]$. This technique may offer a simpler direct route to the nodule but has the potential greater risk of bleeding.

The findings of the study by Fukada and colleagues [13] have practical implications. The use of ultrathin bronchoscopes and injection of physiological saline to dilate the small peripheral airways leading the tumor to allow clearer observation of the endo-luminal features to position forceps or TBNA needles for tissue sampling should lead to a higher diagnostic yield of small peripheral tumors [17].

\section{Conflict of Interest Statement}

The authors have no conflict of interest to declare.

\section{Funding Source}

No funding was received.

\section{Author Contributions}

Dr. Lam and Dr. Shah contribute equally to the paper.

\section{References}

1 National Lung Screening Trial Research Team; Aberle DR, Adams AM, Berg CD, Black WC, Clapp JD, et al. Reduced lung-cancer mortality with low-dose computed tomographic screening. N Engl J Med. 2011;365(5):395-409.

2 De Koning HJ, van der Aalst CM, de Jong PA, Scholten ET, Nackaerts K, Heuvelmans MA, et al. Reduced lung-cancer mortality with volume CT screening in a randomized trial. $\mathrm{N}$ Engl J Med. 2020 Feb 6;382(6):503-13.

3 National Lung Screening Trial Research Team; Church TR, Black WC, Aberle DR, Berg CD, Clingan KL, et al. Results of initial low-dose computed tomographic screening for lung cancer. N Engl J Med. 2013 May 23; 368(21):1980-91.

4 Horeweg N, van Rosmalen J, Heuvelmans MA, van der Aalst CM, Vliegenthart R, Scholten ET, et al. Lung cancer probability in patients with CT-detected pulmonary nodules:

Bronchoscopic Diagnosis of Peripheral Lung Lesions a prespecified analysis of data from the NELSON trial of low-dose CT screening. Lancet Oncol. 2014;15:1332-41.

5 McWilliams A, Tammemagi MC, Mayo JR, Roberts H, Liu G, Soghrati K, et al. Probability of cancer in pulmonary nodules detected on first screening CT. N Engl J Med. 2013; 369(10):910-9.

6 Aberle DR, DeMello S, Berg CD; National Lung Screening Trial Research Team. Results of the two incidence screenings in the National Lung Screening Trial. N Engl J Med. 2013 Sep 5;369(10):920-31.

7 Tammemagi MC, Schmidt H, Martel S, McWilliams A, Goffin JR, Johnston MR, et al. Participant selection for lung cancer screening by risk modelling (the Pan-Canadian Early Detection of Lung Cancer [PanCan] study): a single-arm, prospective study. Lancet Oncol. 2017;18:1523-31.
8 Rivera MP, Mehta AC, Wahidi MM. Establishing the diagnosis of lung cancer: diagnosis and management of lung cancer, 3rd ed: American College of Chest Physicians evidence-based clinical practice guidelines. Chest. 2013;143(5 Suppl):e142S-65S.

9 Sainz Zuñiga PV, Vakil E, Molina S, Bassett R Jr, Ost DE. Sensitivity of radial endobronchial ultrasound guided bronchoscopy for lung cancer in patients with peripheral pulmonary lesions: an updated meta-analysis. Chest. 2020 Apr;157(4):994-1011.

10 McGuire AL, Myers R, Grant K, Lam S, Yee J. The diagnostic accuracy and sensitivity for malignancy of radial-endobronchial ultrasound and electromagnetic navigation bronchoscopy for sampling of peripheral pulmonary lesions: systematic review and metaanalysis. J Bronchology Interv Pulmonol. 2020 Apr;27(2):106-21. 
11 Oki M, Saka H, Asano F, Kitagawa C, Kogure $\mathrm{Y}$, Tsuzuku A, et al. Use of an ultrathin vs thin bronchoscope for peripheral pulmonary lesions: a randomized trial. Chest. 2019 Nov; 156(5):954-64.

12 Chen AC, Pastis NJ, Mahajan AK, Khandhar SJ, Simoff MJ, Machuzak MS, et al. Robotic bronchoscopy for peripheral pulmonary lesions: a Multicenter Pilot and Feasibility Study (BENEFIT). Chest. 2021 Feb;159(2): 845-52.

13 Fukada T, Morita K, Kurimoto N, Setoguchi M, Nosaka S, Katsumata T. The usefulness of a simple classification for bornchoscopic findings for diagnosis of peripheral pulmonary tumor. Respiration. 2021. (MS.Nr: RES2020-6-40/R1 RESUBMISSION).

14 Fielding DIK, Bashirzadeh F, Son JH, Todman M, Chin A, Tan L, et al. First human use of a new robotic-assisted fiber optic sensing navigation system for small peripheral pulmonary nodules. Respiration. 2019;98(2): $142-50$.

15 Herth FJ, Eberhardt R, Sterman D, Silvestri GA, Hoffmann H, Shah PL. Bronchoscopic transparenchymal nodule access (BTPNA): first in human trial of a novel procedure for sampling solitary pulmonary nodules. Thorax. 2015;70(4):326-32.

16 Harzheim D, Sterman D, Shah PL, Eberhardt R, Herth FJF. Bronchoscopic transparenchymal nodule access: feasibility and safety endoscopic unit. Respiration. 2016;91(4):302-6.

17 Naso J, Bras J, Villamil C, Ionescu DN, Wang G, Shaipanich T, et al. Cytologic features and diagnostic value of PeriView FLEX transbronchial needle aspiration targeting pulmonary nodules. Cancer Cytopathol. 2020 May; 128(5):333-40. 